\title{
How should I treat a pulmonary artery rupture occurring during a right heart catheterisation in the cathlab?
}

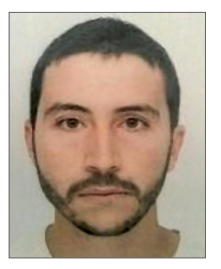

\author{
Madjid Boukantar ${ }^{1 *}$, MD; Romain Gallet ${ }^{1}$, MD; Ketsakim You², MD; Vania Tacher ${ }^{2}$, MD; \\ Gauthier Mouillet ${ }^{1}, \mathrm{MD}$; Hicham Kobeiter ${ }^{2}, \mathrm{MD}$, PhD; Emmanuel Teiger ${ }^{1}$, MD, PhD \\ 1. Henri Mondor Hospital, Explorations Fonctionnelles, Interventional Cardiology, Créteil, France; 2. Henri \\ Mondor Hospital, Imagerie Médicale, Créteil, France
}

Invited experts: Heike A. Hildebrandt ${ }^{3}$, MD; Tienush Rassaf ${ }^{3}$, MD; Philipp Kahlert ${ }^{3}$, MD; Younes Boudjemline ${ }^{4,5}, M D, P h D$ 3. Department of Cardiology and Vascular Medicine, West German Heart and Vascular Center Essen, Essen University Hospital, University Duisburg-Essen, Essen, Germany; 4. Centre de Référence Malformations Cardiaques Congénitales Complexes - M3C, Hôpital Necker Enfants Malades, Assistance Publique des Hôpitaux de Paris, Paris, France; 5. Université Paris Descartes, Paris, France

The concluding section "How did I treat?" together with the complete references and the supplementary data are published online at: http://www.pcronline.com/eurointervention/115th_issue/371

\section{CASE SUMMARY}

BACKGROUND: A 67-year-old woman presenting with symptomatic laminar tricuspid regurgitation was referred to the cathlab for right heart catheterisation. A few seconds after the removal of the Swan-Ganz catheter, a massive haemoptysis appeared, leading to life-threatening respiratory failure.

INVESTIGATION: Clinical assessment, right heart catheterisation, pulmonary angiography.

DIAGNOSIS: Pulmonary artery branch rupture.

MANAGEMENT: A new Swan-Ganz catheter with inflated balloon was advanced in the injured pulmonary artery branch, allowing selective occlusion of the artery and control of the haemoptysis. Then, selective embolisation of the ruptured artery with an AMPLATZER Vascular Plug was performed.

KEYWORDS: AMPLATZER Vascular Plug, pulmonary artery rupture, pulmonary embolisation, Swan-Ganz catheter

\section{PRESENTATION OF THE CASE}

A 67-year-old woman was referred to our cardiology unit for ankle swelling and dyspnoea. Her medical history included a permanent atrial fibrillation treated with warfarin, a moderate tricuspid regurgitation treated with furosemide and a previously treated pulmonary tuberculosis. Her cardiovascular risk factors were arterial hypertension and diabetes mellitus. Clinical examination found signs of chronic right heart failure, an irregular rhythm, and a grade III/VI holosystolic murmur at the lower left sternal border on auscultation. Transthoracic echocardiography (TTE) revealed severe laminar tricuspid regurgitation due to an important annular dilatation, as measured by pulsed wave Doppler (Figure 1). Left ventricular ejection fraction was preserved (55\%) and the tricuspid annular plane systolic excursion (TAPSE) was measured at $18 \mathrm{~cm}$, suggesting preserved right ventricle function. Measurement of pulmonary artery pressure was not obtained because of the laminar tricuspid regurgitation. The case was discussed by our local Heart Team and it was decided that, according to ESC guidelines ${ }^{1}$, surgery (tricuspid annuloplasty) was indicated.

Pre-surgical right heart catheterisation was indicated in order to measure pulmonary artery pressure prior to the heart surgery. It was performed through a $7 \mathrm{Fr}$ right femoral venous access, under local anaesthesia. International normalised ratio was 1.56 the day of the procedure. A Swan-Ganz ${ }^{\mathrm{TM}}$ catheter (Edwards Lifesciences, Irvine, CA, USA) was advanced into the right atrium under X-ray

\footnotetext{
*Corresponding author: Hôpital Henri Mondor, Service des Explorations Fonctionnelles, Cardiologie Interventionnelle, 51 avenue du Maréchal de Lattre de Tassigny,94010 Créteil, France.E-mail: madjid.boukantar@aphp.fr
} 


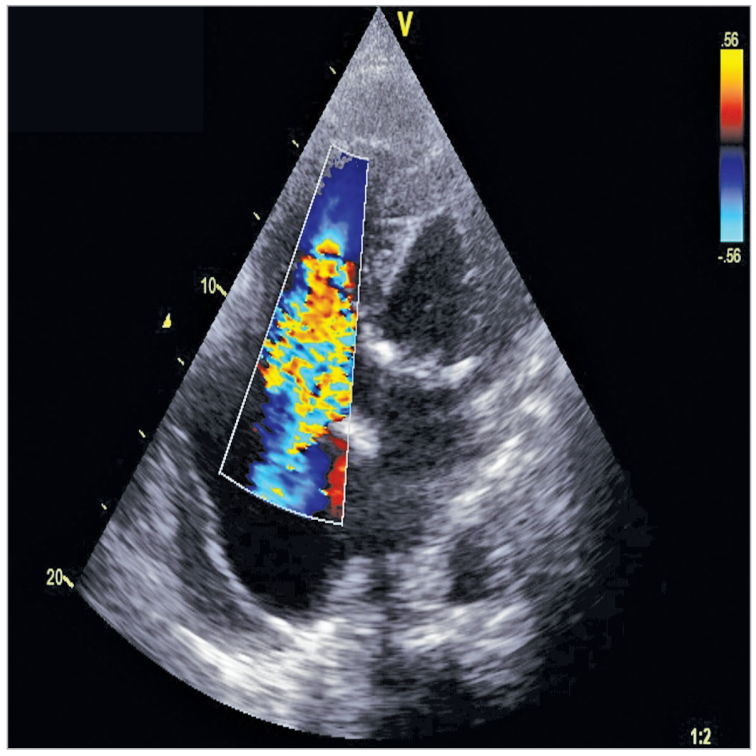

guidance. Then the catheter balloon was inflated and the catheter was pushed until it reached and wedged a branch of the right pulmonary artery. Mean pulmonary wedge pressure was measured at $12 \mathrm{mmHg}$. Other measures performed after balloon deflation included mean pulmonary artery pressure $(30 \mathrm{mmHg})$, right ventricular pressure $(50-4-12 \mathrm{mmHg}$ ) and mean right atrial pressure (12 mmHg). A few seconds after the removal of the Swan-Ganz catheter, the patient started to spit blood. Rapidly, haemoptysis became massive, leading to life-threatening respiratory failure, and pulmonary artery branch rupture due to the Swan-Ganz catheter was diagnosed.

Figure 1. Severe tricuspid regurgitation.

\section{How would I treat?} THE INVITED EXPERTS' OPINION

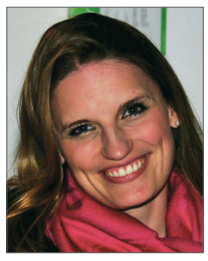

Heike A. Hildebrandt ${ }^{3 *}$, MD; Tienush Rassaf ${ }^{3}$, MD; Philipp Kahlert ${ }^{3}$, MD

3. Department of Cardiology and Vascular Medicine, West German Heart and Vascular Center Essen, Essen University Hospital, University Duisburg-Essen, Essen, Germany

Right heart catheterisation using a Swan-Ganz catheter is a wellestablished tool for the evaluation of valvular heart disease and for haemodynamic monitoring in the intensive care unit. Although right heart catheterisation has proven to be safe, severe and even life-threatening events may occur with an incidence of 0.1 to $0.2 \%$. In fact, pulmonary artery rupture is considered the most dreadful complication, being associated with a mortality rate of approximately $50 \%{ }^{2}$.

Boukantar et al describe such a rare case of pulmonary artery rupture in a 67-year-old patient with severe tricuspid regurgitation, who underwent preoperative right heart catheterisation for haemodynamic evaluation. In the acute setting, patients usually present with cough, acute dyspnoea, haemoptysis, massive haemorrhage, asphyxia and even cardiopulmonary instability, which requires immediate action. After securing an adequate gas exchange and haemodynamic stability (which may even require intubation and bronchoalveolar lavage), the ultimate goal is termination of the bleeding. Here, it has to be considered that surgical therapy by resection of the affected lung section or surgical occlusion of the pulmonary artery's perforation, though effective, is associated with high mortality rates, unless the pulmonary artery perforation occurs during cardiac surgery ${ }^{3}$.

For termination of the bleeding we would follow a stepwise approach based on bleeding severity and size of the laceration. Acute stopping of the bleeding is achieved by immediate repositioning of the Swan-Ganz catheter into the same pulmonary artery segment as before and inflation/wedging of the balloon proximal to the prior location. If the vascular injury is small, this manoeuvre may lead to spontaneous cessation of the bleeding. In addition, thrombin injection (5,000 IU) through the distal lumen of the wedged Swan-Ganz catheter may terminate the bleeding, if the defect is not too large $\mathrm{e}^{4}$.

Larger defects can be visualised by contrast injection over the wedged catheter or an additional pigtail catheter, which is essential for further decision making. Interventional closure of a pulmonary 
artery rupture might be achieved by coil embolisation ${ }^{5}$, as used for occlusions of pulmonary arteriovenous malformations in patients with Osler's disease, or even by implantation of vascular plugs ${ }^{6}$ or covered stents ${ }^{7}$. However, in the emergent situation of an acute pulmonary artery rupture, immediate interventional treatment is limited by the availability of such interventional equipment in adequate sizes and also by the expertise of the interventionalist. Hence, balloon inflation with control of the bleeding is the initial treatment of choice and may buy enough time to organise further escalation of the interventional therapy, if required.

In conclusion, pulmonary artery perforation is a rare but frequently lethal complication that requires carefully balanced deci- sion making between a conservative, interventional and surgical approach. Yet, it should not be forgotten that this dreadful complication can be avoided by some simple rules, specifically (i) do not inflate the balloon blindly and never distal to the main pulmonary artery (always inflate the balloon under pressure-curve guidance until the pulmonary artery curve changes into the wedge curve), (ii) never inflate the balloon against pressure, and (iii) deflate the balloon only while retracting the catheter.

\section{Conflict of interest statement}

The authors have no conflicts of interest to declare.

\section{How would I treat? THE INVITED EXPERT'S OPINION}

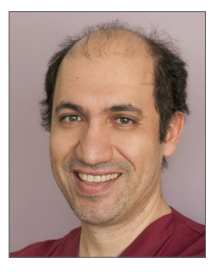

Younes Boudjemline ${ }^{4,5 *}, \mathrm{MD}, \mathrm{PhD}$

4. Centre de Référence Malformations Cardiaques Congénitales Complexes - M3C, Hôpital Necker Enfants Malades, Assistance Publique des Hôpitaux de Paris, Paris, France; 5. Université Paris Descartes, Paris, France

Pulmonary artery rupture can occur following diagnostic or interventional right heart catheterisation. Indications for cardiac catheterisation and wedge pressure measurement should be discussed based on non-invasive assessment. Vessel injury is related to trauma to the pulmonary artery or its branches by the catheter itself, by the wire or by the balloon in the case of balloon dilatation. Quick recognition and treatment are essential to save the patient's life. In parallel to the interventional treatment that is discussed below, adjunctive measures should be taken including calling the anaesthesiologist and cardiac surgeon, resuscitation if needed, administration of intravenous fluid if hypotension ensues, blood transfusion, securing the airways (selective lung intubation in cases with suffocation), and reversing anticoagulation (protamine injection if heparin was given). Interventionists should be prepared and have various devices on the shelf including occlusion devices as well as stents. The type and location of rupture should be precisely diagnosed before attempting any treatment. The interventional treatment to be applied depends largely on the clinical impact and location of the vessel injury. Small bleeding or a contained tear will resolve by itself and can be ignored (after confirming that it remains contained); otherwise, they should be treated aggressively. The rupture of central pulmonary arteries or main branches can be treated using covered stents to force pulmonary flow to pass through the stent and exclude the site of perforation. In distal pulmonary branches as reported by Boukantar et al, I and others favour the use of occlusion devices to occlude the vessel responsible for bleeding. Various devices can be utilised, including AMPLATZERTM Vascular Plugs (Plug I, II or 4; St. Jude Medical, St. Paul, MN, USA), microvascular plugs (MVPTM) or coils (Covidien/Medtronic Neurovascular, Irvine, CA, USA). If the bleeding appeared before removal of the catheter, one could inflate the balloon of the Swan-Ganz catheter, re-advance into the target vessel to stop the bleeding and then use the (wire) lumen of the Swan to advance micro coils such as Concerto $^{\mathrm{TM}}$ coils from Covidien (length depending on the length to occlude). If the bleeding appeared after removal of the Swan catheter, I would rapidly advance a 5 Fr diagnostic catheter into the suspected pulmonary branch and use it to deliver an MVP (MVP 5 , diameter of vessel to occlude up to $5 \mathrm{~mm}$ ) or an AMPLATZER Vascular Plug (Plug 4, $8 \mathrm{~mm}$, diameter of vessel to occlude up to $6 \mathrm{~mm}$ ). In larger vessels, guiding catheters would be needed to deliver devices such as the Vascular Plug II. A final contrast injection is needed to confirm complete occlusion of the culprit pulmonary artery before retrieving the catheter and sheath.

\section{Conflict of interest statement}

Y. Boudjemline is a proctor for St. Jude Medical. 


\section{How did I treat?}

\section{ACTUAL TREATMENT AND MANAGEMENT OF THE CASE}

Management of a pulmonary artery rupture (PAR) is an extreme emergency. Here, the first step was to secure the airways of the patient. Assuming a rupture of a major branch of the pulmonary artery, we immediately paged the intensive care team in order to perform left selective intubation.

The second step was to stop the bleeding. A new Swan-Ganz catheter was advanced until it reached the injured right pulmonary artery branch, allowing selective occlusion of the artery and immediate control of the haemoptysis as diagnosed by the complete cessation of blood reflux from the breathing tube. Selective injection performed through the distal tip of the catheter confirmed the rupture of a major branch with the presence of a pseudoaneurysm

(Figure 2, Moving image 1). The balloon was kept inflated in order to interrupt the feeding of the bleeding artery and to avoid any recurrence of the haemoptysis.

The third step consisted in the control of the haemorrhage. Since the patient was frail, we decided to attempt selective embolisation of the ruptured artery rather than wasting time by moving the patient to the surgical operation room. A 5 Fr sheath was

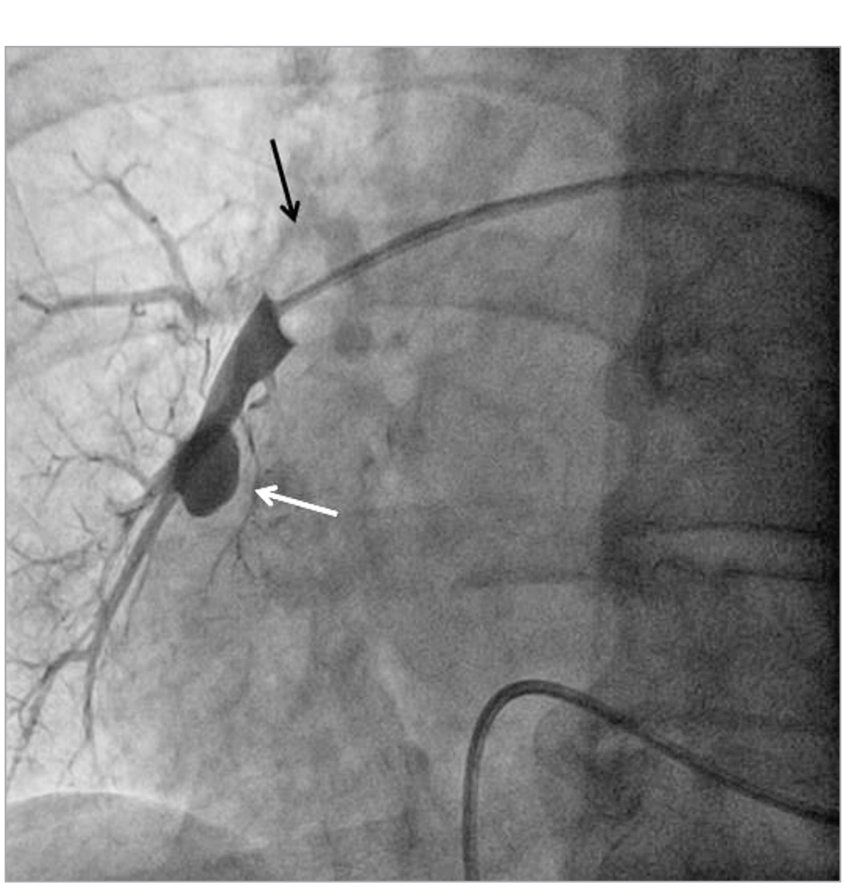

Figure 2. Selective injection at the distal tip of an inflated balloon Swan-Ganz catheter (black arrow) revealing a leak of contrast agent corresponding to a pseudoaneurysm (white arrow). introduced in the left femoral vein. Through this access, a $5 \mathrm{Fr}$ catheter was advanced in the right pulmonary artery, just above the bleeding area. A hydrophilic wire (GLIDEWIRE ADVANTAGE ${ }^{\circledR}$ guidewire; Terumo Corp., Tokyo, Japan) was introduced into the pseudoaneurysm and the catheter was advanced in the pseudoaneurysm over the wire (Figure 3). The wire was then pulled out and an AMPLATZER Vascular Plug 4 of $8 \mathrm{~mm}$ diameter (AVP 4; St. Jude Medical) was introduced into the catheter and deployed in the pseudoaneurysm (Figure 4, Moving image 2). The catheter was then pulled back and a second AVP 4 (5 mm diameter) was deployed in the pulmonary artery branch, just above the origin of the pseudoaneurysm in order to stop the feeding of the bleeding area (Figure 5). Final contrast injection confirmed the occlusion of the pulmonary branch (Figure 6, Moving image 3). The patient was transferred to the intensive care unit with stable haemodynamic and respiratory conditions. She was extubated two days after the procedure and there was no bleeding recurrence. Two months later, she successfully underwent surgical tricuspid annuloplasty associated with left atrial appendage surgical occlusion.

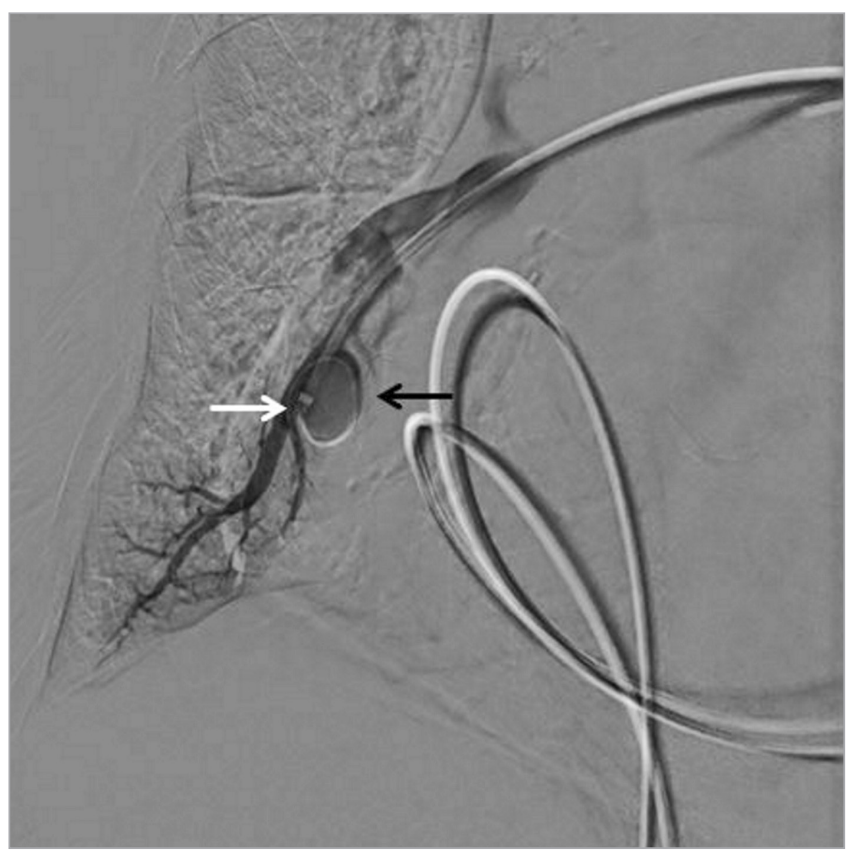

Figure 3. Advancement of a hydrophilic wire rolled in the pseudoaneurysm (black arrow); the tip of the catheter advanced in the pseudoaneurysm through the wire (white arrow). 


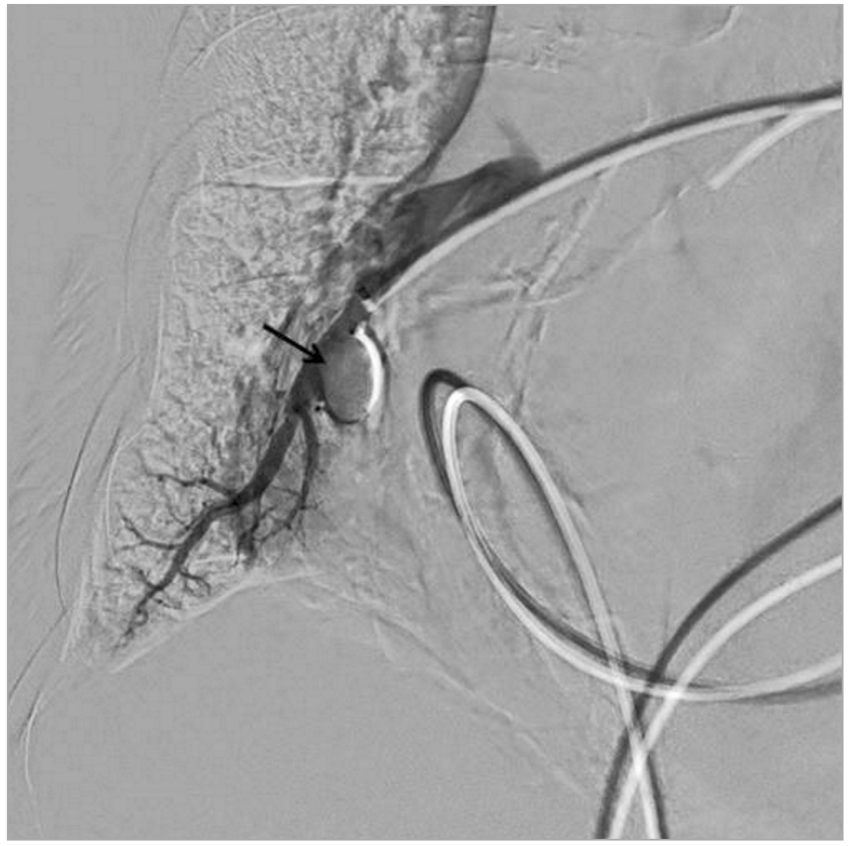

Figure 4. Deployment of an AMPLATZER Vascular Plug 4, $8 \mathrm{~mm}$ in the pseudoaneurysm.

\section{Discussion}

PAR, although rare (incidence of $0.03-0.2 \%$ of all pulmonary artery cannulations) is the most serious Swan-Ganz catheterisation-related complication with a mortality rate as high as $70 \%{ }^{8}$. Classic clinical presentation consists in haemoptysis occurring during the procedure.

Several techniques have been described to manage this lifethreatening complication. The classic technique is the embolisation

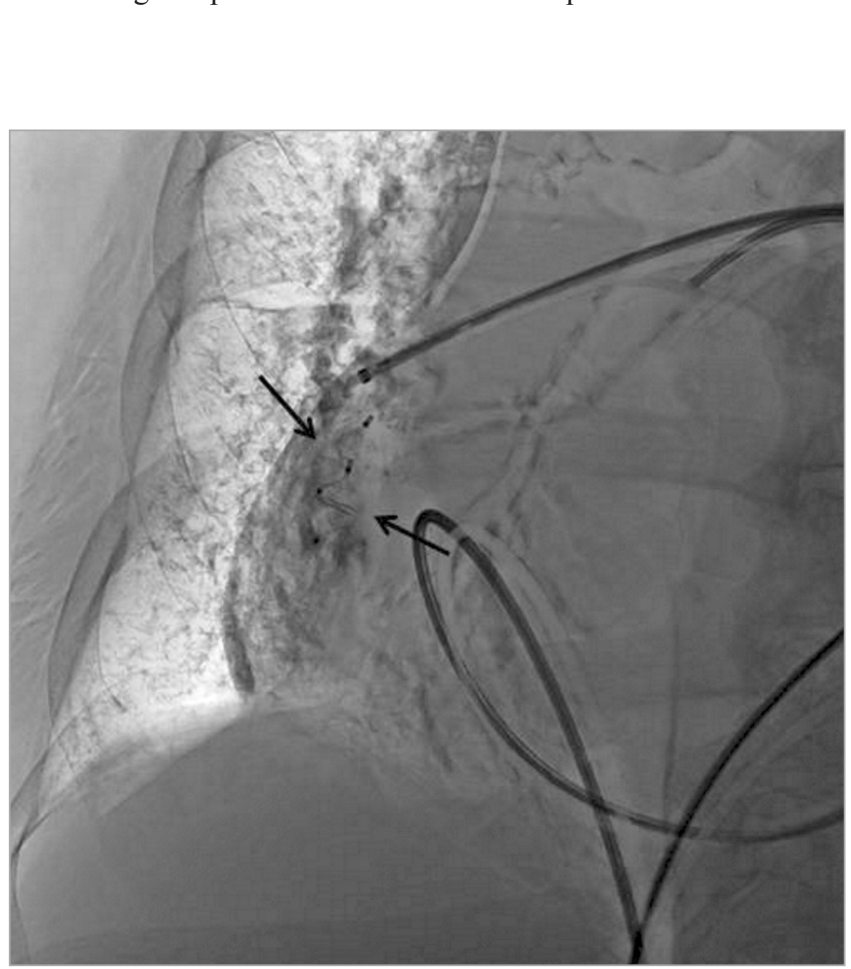

Figure 5. Two AVP 4 devices deployed (black arrows).

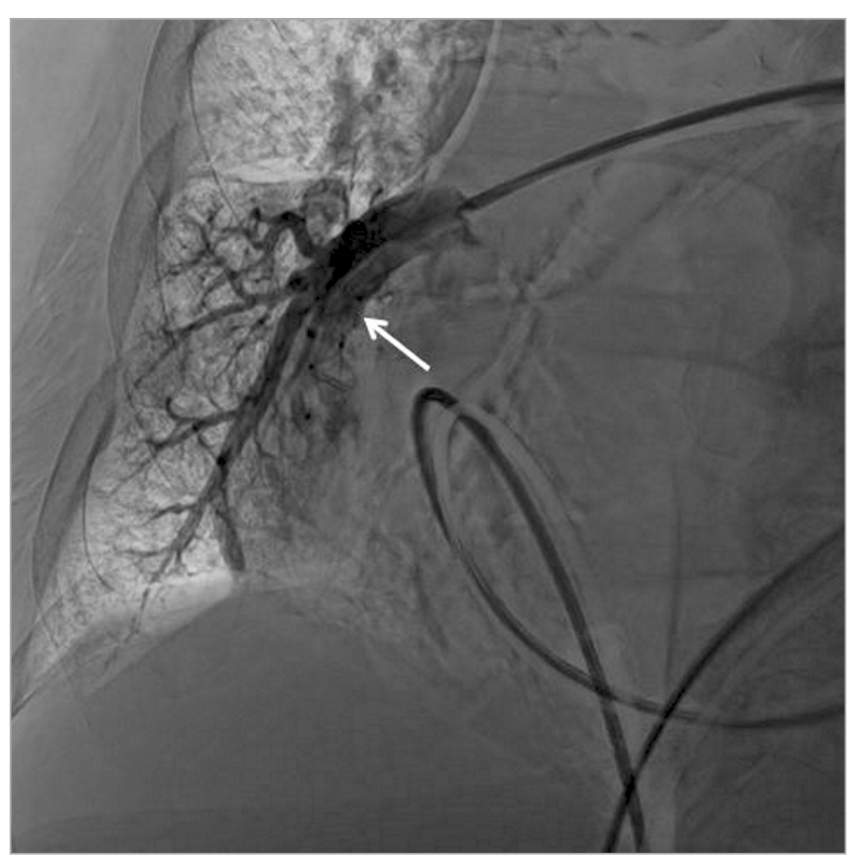

( 0 (2)

Figure 6. Interruption of pulmonary artery blood flow just above the second AVP device (white arrow).

of the bleeding artery by mechanical coils. This technique is expensive when a lot of coils are required, and deployment of coils does not allow optimal control of the rupture, with poor stability and the risk of migration ${ }^{9}$. Selective injection of thrombin in the pseudoaneurysm has been reported. This method seems to be safe but is time-consuming with no guarantee of success ${ }^{10}$. Pulmonary lobectomy can be performed as an emergency life-saving solution when other techniques have failed ${ }^{8}$.

We here describe the technique of percutaneous embolisation with an AMPLATZER device to treat a PAR occurring during right heart catheterisation in the cathlab. The AVP 4 is a 5 Fr catheter-compatible disc made of a mesh of braided nitinol available in 4-8 $\mathrm{mm}$ diameter sizes. Platinum marker bands at both ends of the device make it highly visible under fluoroscopy. The disc is attached to a $155 \mathrm{~cm}$ long, PTFE-coated delivery wire with a stainless steel micro screw, which allows the operator to release the plug into its final position by unscrewing the cable. The plug can be retrieved and readjusted as needed before final release, which provides increased safety during this procedure. The AVP acts as an embolic agent by promoting clot formation. The nitinol mesh induces resistance to blood flow which facilitates fibrin coverage during the clotting process ${ }^{11}$.

\section{Conclusion}

PAR during right heart catheterisation is a rare but life-threatening complication. Early intervention is required to ensure a good outcome. Several endovascular occlusive techniques have been described to ensure adequate haemostasis: coil, thrombin injection, etc. We think that embolisation by an AVP 4 is the best way to treat this complication. This technique allows a quick and conservative 
management of this complication in the cathlab, and is available for unstable patients. The embolisation result can be assessed immediately with a contrast injection, and rapid reintroduction of anticoagulant treatment is safe. The main drawback of this technique is that it may induce a pulmonary infarction although this usually has no consequences.

\section{Conflict of interest statement}

The authors have no conflicts of interest to declare.

\section{References}

1. Joint Task Force on the Management of Valvular Heart Disease of the European Society of Cardiology (ESC), European Association for Cardio-Thoracic Surgery (EACTS), Vahanian A, Alfieri O, Andreotti F, Antunes MJ, Barón-Esquivias G, Baumgartner H, Borger MA, Carrel TP, De Bonis M, Evangelista A, Falk V, Iung B, Lancellotti P, Pierard L, Price S, Schäfers HJ, Schuler G, Stepinska J, Swedberg K, Takkenberg J, Von Oppell UO, Windecker S, Zamorano JL, Zembala M. Guidelines on the management of valvular heart disease (version 2012). Eur Heart J. 2012;33:2451-96.

2. Utsumi $T$, Kido $T$, Ohata $T$, Yasukawa $M$, Takano $H$, Sakakibara T. Swan-Ganz catheter-induced pseudoaneurysm of the pulmonary artery. Jpn J Thorac Cardiovasc Surg. 2002;50:347-9.

3. Bossert T, Gummert JF, Bittner HB, Barten M, Walther T, Falk V, Mohr FW. Swan-Ganz catheter-induced severe complications in cardiac surgery: right ventricular perforation, knotting, and rupture of a pulmonary artery. J Card Surg. 2006;21:292-5.

4. Sack S, Kahlert P, Philipp S, Kottenberg-Assenmacher E, Churzidse S, Konorza T, Peters J, Erbel R. Reocclusion with thrombin after perforation of the peripheral pulmonary artery with haemorrhage during right heart catheterization. Intensivmedizin und Notfallmedizin. 2007;44:520-25.
5. Rudziński PN, Henzel J, Dzielińska Z, Lubiszewska M, Michalowska I, Szymański P, Pracoń R, Hryniewiecki T, Demkow M. Pulmonary artery rupture as a complication of SwanGanz catheter application. Diagnosis and endovascular treatment: a single centre's experience. Postepy Kardiol Interwencyjnej. 2016;12:135-9.

6. Ianniello A, Carrafiello G, Nicotera P, Vaghi A, Cazzulani A. Endovascular treatment of a ruptured pulmonary artery aneurysm in a patient with Behçet's disease using the Amplatzer Vascular Plug 4. Korean J Radiol. 2013;14:283-6.

7. Ejiri K, Ogawa A, Matsubara H. Bail-out technique for pulmonary artery rupture with a covered stent in balloon pulmonary angioplasty for chronic thromboembolic pulmonary hypertension. JACC Cardiovasc Interv. 2015;8:752-3.

8. Kearney TJ, Shabot MM. Pulmonary artery rupture associated with the Swan-Ganz catheter. Chest. 1995;108:1349-52.

9. Poplausky MR, Rozenblit G, Rundback JH, Crea G, Maddineni S, Leonardo R. Swan-Ganz catheter-induced pulmonary artery pseudoaneurysm formation: three case reports and a review of the literature. Chest. 2001;120:2105-11.

10. Dobies DR, Cohoon AL, Bates AA. Repair of a perforated pulmonary artery due to a Swan-Ganz catheter using thrombin injection. Circulation. 2009;119:e521-2.

11. Cil B, Peynircioğlu B, Canyiğit M, Geyik S, Ciftçi T. Peripheral vascular applications of the Amplatzer vascular plug. Diagn Interv Radiol. 2008;14:35-9.

\section{Supplementary data}

Moving image 1. Tip injection showing false aneurysm.

Moving image 2. Deployment of an AVP 4 device in the false aneurysm.

Moving image 3. Final injection showing exclusion of the bleeding artery. 